\title{
Rentabilidad del estudio citológico del líquido pleural en el derrame maligno
}

\author{
S. BIELSA, M. J. PANADÉS ${ }^{1}$, R. EGIDO ${ }^{1}$, M. RUE ${ }^{2}$, A. SALUD ${ }^{3}$, X. MATÍAS-GUIU ${ }^{1}$, \\ F. RODRÍGUEZ-PANADERO ${ }^{4}$, J. M. PORCEL
}

Servicio de Medicina Interna. IRBLLEIDA. ${ }^{~}$ Servicio de Anatomía Patológica. ${ }^{2}$ Departamento de Ciencias Médicas Básicas. Universidad de Lleida. ${ }^{3}$ Servicio de Oncología Médica. Hospital Universitario Arnau de Vilanova. Lleida. ${ }^{4}$ Unidad MédicoQuirúrgica de Enfermedades Respiratorias. Hospital Virgen del Rocío. Sevilla

\author{
ACCURACY OF PLEURAL FLUID CYTOLOGY IN MALIGNANT \\ EFFUSIONS
}

\section{RESUMEN}

Objetivo: Evaluar la rentabilidad de los estudios citológicos sucesivos del líquido pleural (LP) para diagnosticar malignidad y analizar la influencia que sobre aquella tienen el tiempo transcurrido entre los análisis, el tamaño del derrame y las características bioquímicas del LP.

Métodos: Se revisaron retrospectivamente 1.427 pacientes con derrame pleural (DP), de los que 466 eran de causa maligna. En este último grupo se analizaron las citologías sucesivas, el tiempo transcurrido entre las mismas, las características bioquímicas del LP y el tamaño del DP.

Resultados: La sensibilidad de una primera citología fue del 48,5\%. Cuando un primer estudio citológico era negativo, un segundo era diagnóstico en el 28,6\% de los casos, mientras que con dos citologías negativas un tercer estudio conseguía un $10,3 \%$ de positividades adicionales. El tipo de tumor condiciona la rentabilidad de la citología (66,5\% en adenocarcinomas frente a $30,8 \%$ en mesoteliomas), pero no así el tiempo transcurrido entre los análisis citológicos sucesivos ni el tamaño del DP. De los parámetros bioquímicos del LP, un análisis multivariante mostró que sólo un cociente entre la glucosa del LP y del suero $\leq 0,75$ se relacionaba con una mayor sensibilidad de la citología ( $74 v s .47 \%, \mathrm{p}<0,001)$.

Conclusión: Se aconseja repetir al menos una segunda citología en todo DP de etiología incierta, cuando una primera ha resultado negativa. Este segundo estudio se puede realizar de forma inmediata ya que el paso del tiempo no incrementa la rentabilidad. El porcentaje de positividades está influido por el tipo de tumor y por algunas características bioquímicas del LP, como el cociente entre la glucosa del LP y del suero.

PALABRAS CLAVE: Derrame pleural. Derrame pleural maligno. Citología. Líquido pleural.

\section{ABSTRACT}

Objective: To assess the usefulness of repeat cytological examination of pleural fluid $(P F)$ for diagnosing malignancy as well as the influence of time length between analyses, effusion's size and pleural fluid biochemistries on the diagnostic yield of cytology.

Methods: Retrospective analysis of 1,427 patients with pleural effusion (PE), including 466 patients with malignant PE. In this latter group, the time length between cytological analysis, the size of the PE, and the biochemical characteristics of $P F$ were recorded.

Results: The first cytological analysis had a sensitivity of $48.5 \%$. If this was negative, a second PF specimen was diagnostic in $28.6 \%$ of cases, whereas submission of a third PF specimen allowed $10.3 \%$ of additional diagnosis. The incidence of positive results depended on the primary tumor (e.g. $66.5 \%$ in adenocarcinomas, $30.8 \%$ in mesotheliomas), but neither on the time length between cytological analyses nor on the effusion's size. A multivariate analysis showed that a PF to serum glucose ratio $\leq 0.75$ was associated with a higher diagnostic yield of cytology $(74 v s .47 \%, p<0.001)$.

Conclusion: At least a second PF specimen should be submitted immediately for cytologic analyis in all PE of unknown cause, when the first analysis is not contributory. To delay this second analysis does not increase diagnostic yield. The percentage of cases in which cytologic study of the PF established the diagnosis of malignant PE depends on the tumor type and on certain $P F$ biochemical characteristics such as the $P F$ to serum glucose ratio.

KEY WORDS: Pleural effusion. Malignant pleural effusion. Cytology. Pleural fluid.

Bielsa S, Panadés MJ, Egido R, Rue M, Salud A, Matías-Guiu X, Rodríguez-Panadero F, Porcel JM. Rentabilidad del estudio citológico pleural en el derrame maligno. An Med Interna (Madrid) 2008; 25: 173-177.

\section{INTRODUCCIÓN}

La causa más frecuente de derrame pleural (DP) entre los pacientes que se someten a una toracocentesis es el cáncer (1). En una serie necrópsica se halló DP en 30 (16\%) de 191 pacientes con cáncer (2). Los tumores que con mayor frecuencia se extienden a la superficie pleural son el cáncer de pulmón y el de mama, que en conjunto son responsables de aproximadamente la mitad de todos los DPs malignos (DPM) (3).
El estudio citológico del líquido pleural (LP) es el método más utilizado, por su simplicidad, para diagnosticar el DPM. La biopsia pleural a ciegas tiene menor rentabilidad que la citología (4), mientras que la toracoscopia permite asegurar la naturaleza maligna de un DP en más del 95\% de los casos (5).

La bibliografía médica describe una gran variación tanto en la sensibilidad global de la citología pleural para diagnosticar malignidad, que oscila entre el 40 y el 87\% (6-8), como en el incremento porcentual de sensibilidad de los estudios cito- 
lógicos sucesivos (de 7 a $55 \%$ para segundas citologías $(9,10)$ y de 6 a $62 \%$ para terceras citologías) $(10,11)$. Sin embargo, con referencia a este último dato se desconoce la influencia que puede tener el tiempo transcurrido entre la obtención de una y otra muestra de LP. Además, existen trabajos contradictorios respecto a las variables del LP que se asocian con una mayor rentabilidad de la citología en el DPM. Así, mientras algunos estudios describen una mayor sensibilidad de la citología en pacientes con concentraciones pleurales bajas de glucosa y pH $(12,13)$, otros no confirman este extremo (14).

Los objetivos de este estudio, realizado sobre un gran número de pacientes con DPM, fueron: a) conocer la sensibilidad de los estudios citológicos sucesivos y la influencia del tiempo transcurrido entre ellos para diagnosticar malignidad; y b) averiguar si factores como el tamaño del DP o las características bioquímicas del LP, predicen un mayor porcentaje de positividad de la citología.

\section{PACIENTES Y MÉTODOS}

Hemos revisado retrospectivamente la base de datos de DPs de nuestro hospital, donde se recogen las características clínicas, radiológicas y de laboratorio de todos los pacientes con DP a los que se realiza una toracocentesis desde junio de 1993. Para el presente estudio se incluyeron todos los pacientes con al menos un estudio citológico del LP desde esa fecha hasta julio de 2006. La decisión de realizar citologías adicionales del LP dependió del criterio del clínico responsable.

De cada paciente, se recogieron los siguientes datos: edad y sexo; tamaño y localización del DP en una proyección radiológica postero-anterior; datos bioquímicos del LP (recuento celular total y diferencial, glucosa, proteínas, lactatodeshidrogenasa -LDH-, adenosina desaminasa -ADA-, amilasa y $\mathrm{pH}$ ) y de la sangre (glucosa, proteínas y LDH); citologías del LP y su fecha de realización; biopsia pleural si se había efectuado; diagnóstico final, y si éste era de DPM, el origen del tumor primario y su histología.

\section{ANÁLISIS BIOQUÍMICO Y CITOLÓGICO DEL LP}

El LP obtenido mediante toracocentesis se repartió en tubos estériles de $5 \mathrm{~mL}$ que contenían heparina para su análisis bioquímico y citológico. La medición de las concentraciones de glucosa, proteínas, LDH, colesterol, amilasa y ADA se realizó en un autoanalizador Hitachi (modelos 717 ó 917) por métodos estandarizados y la de $\mathrm{pH}$ en un analizador de gasometrías. El recuento celular se realizó manualmente en una cámara de Thoma. En nuestro hospital el límite superior de la normalidad para la LDH sérica es de 470 U/l, y el de amilasa de 100 U/l. Las muestras de LP que se remitieron al laboratorio de anatomía patológica se centrifugaron durante 10 minutos a 3.000 revoluciones por minuto, tiñéndose el botón celular con la técnica de Papanicolau y extendiéndose en dos laminillas para su examen microscópico. Todas las muestras se reexaminaron por dos patólogos de forma independiente, que no disponían de información clínica del paciente. Las discrepancias en el diagnóstico citológico se resolvieron por consenso después de una revisión conjunta entre ambos.

Las citologías se clasificaron como "positivas", "negativas" o "sospechosas". Se consideraron negativas si se objetivaban células inflamatorias o mesoteliales reactivas y positivas si exis- tían células con rasgos atípicos manifiestos. El término citología sospechosa, basado en una apreciación subjetiva del patólogo, se aplicó cuando existían características citológicas muy sugestivas, pero no inequívocas de malignidad.

\section{CRITERIOS DIAGNÓSTICOS}

Los pacientes con DP se clasificaron en tres grupos etiológicos: DPM definitivo, DP probablemente maligno y DP benigno. Un DPM exigía la demostración de células malignas en la citología o en la biopsia pleural. Aquellos pacientes con un cáncer conocido y un DP persistente hasta el fallecimiento (salvo que se realizara pleurodesis), con citología del LP negativa, y sin otra causa aparente como infecciones, insuficiencia cardiaca, atelectasia o tromboembolismo pulmonar se consideraron portadores de un DP probablemente maligno. En este sentido, si existían dudas diagnósticas o no se habían realizado las exploraciones complementarias oportunas para descartar causas alternativas del DP, el paciente se excluyó del estudio. Finalmente, el diagnóstico de los DPs benignos se basó en criterios clínicos universalmente aceptados (15).

Se clasificó a un DP como grande cuando ocupaba la mitad o más del hemitórax en una proyección radiológica postero-anterior y masivo si lo hacía de forma completa.

\section{ANÁLISIS ESTADÍSTICO}

Los intervalos de confianza de las proporciones se estimaron utilizando el método exacto de la distribución binomial. Para evaluar la asociación entre variables cualitativas se utilizó el test de Chi-cuadrado y para evaluar la influencia de variables del paciente en la rentabilidad de la citología, ajustado por otras variables, se utilizó el análisis de regresión logística.

Para el análisis de la sensibilidad de la citología se consideraron las citologías sospechosas como diagnósticas de malignidad, salvo que se indique lo contrario. Para conocer la influencia del tiempo en la rentabibilidad de la citología, los pacientes con más de una citología se clasificaron en cuartiles según los días transcurridos entre el análisis de una y otra muestra de LP. Se calculó la sensibilidad de la citología para diagnosticar malignidad dentro de cada cuartil y se compararon las diferencias mediante una prueba de regresión logística binaria.

Finalmente, para estudiar la influencia de los parámetros bioquímicos sobre la rentabilidad de la citología, se seleccionaron puntos de corte mediante curvas de eficacia diagnóstica (ROC), buscando una especificidad del 90\%. La significación estadística se estableció para una $\mathrm{p} \leq 0,05$. Los análisis se realizaron con el paquete estadístico SPSS versión 11.5 (Chicago, IL, USA).

\section{RESULTADOS}

\section{PACIENTES Y TIPOS DE TUMOR}

Se analizaron las citologías de 1427 pacientes con DP, de los cuales 466 tenían un DPM. De este último grupo, todos disponían de al menos uno, 119 de dos y 29 de tres estudios citológicos del LP. Existían 284 pacientes con DPM definitivo (263 confirmados por citología y 21 por biopsia pleural) y 182 con 


\begin{tabular}{lccc}
\multicolumn{4}{c}{ TABLA I } \\
\multicolumn{4}{c}{ ORIGEN DEL TUMOR EN LOS DERRAMES PLEURALES } \\
MALIGNOS \\
\hline Tumor \\
priario & $n(\%)$ & $\begin{array}{c}\text { Derrame maligno } \\
\text { definitivo }\end{array}$ & $\begin{array}{c}\text { Derrame maligno } \\
\text { probable }\end{array}$ \\
\hline Pulmón & & 97 & 70 \\
Adenocarcinoma & $167(35,8)$ & 59 & 13 \\
Escamoso & $72(15,4)$ & 12 & 24 \\
Microcítico & $36(7,7)$ & 10 & 15 \\
Otros & $25(5,4)$ & 16 & 17 \\
Mama & $33(7,1)$ & 57 & 26 \\
Desconocido & $83(17,8)$ & 29 & 12 \\
Linfoma $_{\text {Ovario }}$ & $41(8,8)$ & 24 & 14 \\
Mesotelioma $_{\text {Castrointestinal }}^{*}$ & $38(8,1)$ & 23 & 12 \\
Otros $^{\dagger}$ & $35(7,5)$ & 12 & 1 \\
Total $^{*}$ & $31(6,8)$ & 11 & 20 \\
\hline
\end{tabular}

*: 3 esófago, 16 colorrectal, 12 estómago; t: 9 hipernefroma, 9 cabezacuello, 5 melanoma, 5 endometrio, 4 próstata, 4 síndrome mielodisplásico, 3 leucemia linfática crónica, 3 timoma, 3 mieloma, 2 cérvix, 2 leucemia aguda linfoide, 2 sarcoma partes blandas, 1 tiroides, 1 colangiocarcinoma, 1 páncreas, 1 suprarrenal, 1 sarcoma pleural, 1 vulva, 1 mulleriano uterino, 1 macroglobulinemia de Waldenstrom.

DP probablemente maligno. Se trataba de 247 varones y 219 mujeres con una edad media (desviación estándar) de 67 (13) años. Como se observa en la tabla I, el tipo de tumor primario que con mayor frecuencia afectó a la pleura fue el cáncer de pulmón $(35,8 \%)$, seguido del cáncer de mama $(17,8 \%)$, cáncer de origen desconocido $(8,8 \%)$, linfoma $(8,1 \%)$ y ovario $(7,5 \%)$. Con respecto al tipo histológico, se incluyeron las siguientes categorías: adenocarcinomas (270), leucemias y linfomas (51), carcinomas escamosos (49), carcinomas de célula pequeña (25), mesoteliomas (13), sarcomas (5) y miscelánea (53).

\section{RENTABILIDAD DE LOS ESTUDIOS CITOLÓGICOS SUCESIVOS}

Se evaluaron 614 citologías pleurales pertenecientes a 466 pacientes con DPM. El primer estudio citológico fue positivo en 226 pacientes $(48,5 \%$, IC95\% 43,9-53,1\%). De 119 pacientes en los que una primera citología fue negativa, un segundo estudio consiguió diagnosticar a 34 (28,6\%, IC95\% 20,7$37,6 \%$ ). De 29 pacientes en los que dos citologías sucesivas resultaron negativas, una tercera dio resultados positivos en sólo 3 casos (10,3\%, IC95\% 2,2-27,4\%). Por otra parte, de 20 pacientes en los que la primera citología se consideró sospechosa, la segunda resultó positiva en 19 (95\%, IC 95\% 75,1\%$100 \%)$. La sensibilidad global de la citología en el diagnóstico del DPM fue de 56,4\% (IC95\% 51,9-60,9\%).

Para analizar la influencia del tiempo sobre la sensibilidad de las citologías sucesivas, se consideraron dos grupos: un primero constituido por aquellos pacientes en los que una nueva citología continuaba siendo negativa y un segundo formado por los pacientes en los que una segunda o tercera citologías cambiaban de negativa a positiva o sospechosa. La mediana (rango intercuartílico) de tiempo entre los estu-
TABLA II

\begin{tabular}{|c|c|c|c|c|}
\hline \multicolumn{5}{|c|}{$\begin{array}{c}\text { INFLUENCIA DEL TIEMPO EN LA RENTABILIDAD DE } \\
\text { CITOLOGÍAS SUCESIVAS }\end{array}$} \\
\hline & $\begin{array}{c}N^{0} \text { de } \\
\text { pacientes }\end{array}$ & $\begin{array}{l}\text { Días transcurridos entre } \\
\text { los estudios citológicos } \\
\text { (mediana } \pm \text { rango } \\
\text { intercuartílico) }\end{array}$ & $\begin{array}{c}\text { Sensibilidad de la } \\
\text { citología (con una } \\
\text { previa negativa) }\end{array}$ & $p^{*}$ \\
\hline $1^{\circ}$ cuartil & 48 & $1 \pm 0$ & $31 \%$ & 0,95 \\
\hline $2^{\circ}$ cuartil & 30 & $3 \pm 2$ & $27 \%$ & \\
\hline $3^{\circ}$ cuartil & 42 & $7 \pm 4$ & $31 \%$ & \\
\hline $4^{\circ}$ cuartil & 42 & $36,5 \pm 84$ & $33 \%$ & \\
\hline Global & 162 & $5 \pm 14$ & $31 \%$ & \\
\hline
\end{tabular}

p* obtenida a partir de la realización de una regresión logística binaria.

dios citológicos fue de 5 (14) días en ambos grupos. Como se observa en la tabla II, el número de días transcurridos entre los análisis citológicos, desglosado en cuartiles, no influyó en la rentabilidad de la citología.

\section{RENTABILIDAD DE LA CITOLOGÍA SEGÚN EL TIPO DE TUMOR}

La sensibilidad global de la citología varió según la estirpe histológica del tumor primario: adenocarcinomas 66,5\% (IC95\% $60,9-72,2 \%$ ), linfomas 54,9\% (IC95\% 40,8-69\%), carcinomas de célula pequeña 40\% (IC95\% 19,4-60,6\%), carcinomas escamosos 34,7\% (IC95\% 20,9-48,5\%) y mesoteliomas 30,8\% (IC95\% $1,7-59,8 \%)$. En ninguno de los 5 sarcomas se obtuvo una citología positiva.

\section{BIOQUÍMICA DEL LP, TAMAÑO DEL DERRAME Y RENTABILIDAD DE LA CITOLOGÍA}

El análisis ROC permitió establecer los siguientes puntos de corte de los parámetros bioquímicos del LP, capaces de predecir la positividad de una citología con una especificidad del 90\%: $\mathrm{pH} \leq 7,3$, glucosa $\leq 75 \mathrm{mg} / \mathrm{dl}$, cociente de glucosa entre $\mathrm{LP}$ y suero $\leq 0,75, \mathrm{LDH} \geq 1.500 \mathrm{U} / 1$, proteínas $\leq 52 \mathrm{~g} / \mathrm{l}$, cociente de proteínas entre LP y suero $\leq 0,77$, hematíes $\geq 3 \mathrm{x}$ $10 \%$, leucocitos $\geq 0,4 \times 10^{9} / 1$, porcentaje de neutrófilos $\leq$ $10 \%, \mathrm{ADA} \geq 30 \mathrm{U} / \mathrm{l}$, colesterol $\geq 100 \mathrm{mg} / \mathrm{dl}$ y amilasa $\geq 90$ U/L. De este modo, la citología fue más rentable en los DPMs con cifras de $\mathrm{pH} \leq 7,3$ (71 vs. 54\%, $\mathrm{p}<0,01)$, un cociente de glucosa LP/suero $\leq 0,75$ (74 vs. 47\%, p < 0,001), una LDH $\geq$ $1.500 \mathrm{U} / 1$ (68 vs. 54\%, p <0,04), un cociente de proteínas $\mathrm{LP} /$ suero $\leq 0,77$ (70 vs. 54\%, $\mathrm{p}<0,02)$ y una amilasa $\geq 90 \mathrm{U} / 1$ (75 vs. 47\%, p <0,02). Quedaron al límite de la significación estadística los LPs con una concentración de glucosa $\leq 75$ $\mathrm{mg} / \mathrm{dl}(72 v s .54 \% \mathrm{p}=0,06)$. Por último, variables como las proteínas, el recuento de hematíes o leucocitos, el porcentaje de neutrófilos, la ADA y el colesterol con los puntos de corte arriba señalados, así como el tamaño radiológico del DP no influyeron en la sensibilidad de la citología.

Se realizó un análisis multivariante donde se incluyeron las variables significativas expresadas en la tabla III, excepto la amilasa debido al escaso número de pacientes con esta determinación. Al ajustar un modelo de regresión logística se 
TABLA III

\section{SENSIBILIDAD DE LA CITOLOGÍA SEGÚN EL TAMAÑO DEL DERRAME Y LAS CARACTERÍSTICAS BIOQUÍMICAS DEL LÍQUIDO PLEURAL}

\begin{tabular}{|c|c|c|c|c|}
\hline Variables & Valores & $n(\%)$ & $\begin{array}{c}\text { Sensibilidad citológica } \\
\text { (IC95\%) }\end{array}$ & $p^{*}$ \\
\hline \multirow[t]{4}{*}{ Tamaño del DP } & $<1 / 2$ & $144(36)$ & $51(43-57)$ & 0,17 \\
\hline & $\geq 1 / 2$ & $253(64)$ & $58(52-62)$ & \\
\hline & No masivo & $314(79)$ & $55(49-60)$ & 0,37 \\
\hline & Masivo & $83(21)$ & 60 (49-71) & \\
\hline \multirow[t]{2}{*}{$\mathrm{pH}$} & $\leq 7,3$ & $58(15)$ & $71(59-83)$ & 0,01 \\
\hline & $>7,3$ & $336(85)$ & $54(48-59)$ & \\
\hline \multirow[t]{2}{*}{ Clucosa (mg/dl) } & $\leq 75$ & $67(15)$ & $72(61-83)$ & 0,06 \\
\hline & $>75$ & $393(85)$ & $54(49-59)$ & \\
\hline \multirow[t]{2}{*}{ Clucosa LP/suero } & $\leq 0,75$ & $54(18)$ & $74(62-86)$ & 0,001 \\
\hline & $>0,75$ & $252(82)$ & $47(41-53)$ & \\
\hline \multirow[t]{2}{*}{ LDH (UI/l) } & $<1.500$ & $393(86)$ & $54(50-59)$ & 0,04 \\
\hline & $\geq 1500$ & $63(14)$ & $68(56-80)$ & \\
\hline \multirow[t]{2}{*}{ Amilasa (UI/I) } & $<90$ & $93(82)$ & $47(37-58)$ & 0,02 \\
\hline & $\geq 90$ & $20(18)$ & $75(54-96)$ & \\
\hline \multirow[t]{2}{*}{ Proteínas (g/l) } & $\leq 52$ & 401 (87) & $56(51-60)$ & 0,4 \\
\hline & $>52$ & $59(13)$ & $61(48-74)$ & \\
\hline \multirow[t]{2}{*}{ Proteínas LP/suero } & $\leq 0,77$ & $353(86)$ & $54(48-59)$ & 0,02 \\
\hline & $>0,77$ & $56(14)$ & $70(57-82)$ & \\
\hline \multirow[t]{2}{*}{ Hematíes (x 109/l) } & $<3$ & $174(38)$ & $53(45-60)$ & 0,2 \\
\hline & $\geq 3$ & $281(62)$ & $59(53-65)$ & \\
\hline \multirow[t]{2}{*}{ Leucocitos (x 10\%/l) } & $<0,4$ & $107(23)$ & $58(48-67)$ & 0,7 \\
\hline & $\geq 0,4$ & $349(77)$ & $56(51-61)$ & \\
\hline \multirow[t]{2}{*}{ Neutrófilos (\%) } & $\leq 10$ & $160(38)$ & $53(45-61)$ & 0,4 \\
\hline & $>10$ & $260(62)$ & $57(51-63)$ & \\
\hline \multirow[t]{2}{*}{ ADA (U/I) } & $<30$ & $383(86)$ & $57(52-62)$ & 0,5 \\
\hline & $\geq 30$ & $28(6)$ & $53(40-66)$ & \\
\hline \multirow[t]{2}{*}{ Colesterol (mg/dl) } & $<100$ & $85(74)$ & $64(53-74)$ & 0,5 \\
\hline & $\geq 100$ & $30(26)$ & $70(53-87)$ & \\
\hline
\end{tabular}

*Valor p de la prueba Chi-cuadrado (análisis univariante); ADA: adenosina desaminasa; DP: derrame pleural; LDH: lactato deshidrogenasa; LP: líquido pleural.

observó significación estadística sólo para el cociente de glucosa entre LP y suero $\leq 0,75$ (odds ratio 3; IC95\% 1,3-7,1).

\section{DISCUSIÓN}

La citología del LP constituye una exploración obligada en el estudio de cualquier DP de etiología desconocida (16), ya que permite confirmar la existencia de malignidad de una forma sencilla y rápida. En este estudio la sensibilidad global de la citología para diagnosticar malignidad fue cercana al $60 \%$, acorde con lo descrito por otros autores (17). Se debe tener en cuenta que en algunos pacientes con cáncer, el DP no se relaciona directamente con la infiltración tumoral de la pleura y por tanto no se puede esperar encontrar células tumorales en el LP. Efectos locales (obstrucción linfática, atelectasia, pulmón atrapado) o sistémicos (embolia pulmonar, hipoalbuminemia) del tumor o complicaciones de la terapia utilizada explican el desarrollo de estos DP "paramalignos" (18), que razonablemente fueron excluidos en nuestra población de estudio. No obstante, en un porcentaje significativo de pacientes con DPM se observan citologías falsamente negativas. A este hecho contribuyen diversos factores como la extensión de la enfermedad, el tipo de neoplasia primaria, la experiencia del patólogo, la calidad y preservación de las células, así como el tipo (extensiones vs bloques celulares) y el número de especimenes examinados $(7,11)$. Otros factores antaño considerados determinantes, como el volumen de LP analizado, no se han confirmado en investigaciones recientes. Así, Sallach et al demostraron que un volumen de unos $5 \mathrm{ml}$, como el utilizado en nuestro estudio, es tan eficaz como volúmenes de hasta $1.000 \mathrm{ml}$ para identificar células malignas (19).

Los resultados de este trabajo muestran que la sensibilidad de la citología en los adenocarcinomas metastáticos $(66,5 \%)$ duplica la hallada para los mesoteliomas $(30,8 \%)$, mientras que los linfomas se sitúan en unas cifras intermedias $(54,9 \%)$, hallazgos que corroboran descripciones previas $(20,21)$.

La repetición del análisis citológico aumenta la probabilidad de encontrar un resultado positivo cuando el DP es maligno, entre un $7 \%$ y un 55\% (4,9-11,22-25). En nuestra serie, la que dispone del mayor número de pacientes con citologías repetidas, este porcentaje fue del 28,6\%. Diagnosticar casi un tercio de DPMs adicionales permite recomendar la obtención de una segunda citología en caso de que la primera sea negativa. Sin embargo, cuando dos estudios citológicos han sido negativos una nueva muestra será diagnóstica en el 6 al $62 \%$ de los casos, según la bibliografía médica $(9-11,17,22-24)$, y en el 10,3\% según nuestros resultados, por lo que la conveniencia de una tercera toracocentesis debería dejarse al criterio personal de cada médico.

En este estudio demostramos por primera vez que el diagnóstico de malignidad basado en la citología no depende del momento en que ésta se realice, circunstancia no referenciada en investigaciones previas. Es decir, se debería obtener de forma inmediata una segunda muestra de LP para el análisis citológico cuando una primera citología ha sido negativa y se sospecha malignidad. La demora en obtener un segundo estudio citológico, con la hipótesis de que el progreso de la enfermedad podría aumentar la rentabilidad citológica, no estaría justificada.

Otro aspecto de nuestra investigación fue conocer si el tamaño del DP o algún parámetro bioquímico del LP influyen en la rentabilidad de la citología. En cuanto al primero, si bien Jiménez y cols., (26) describieron mayor sensibilidad de la citología en DPMs masivos (57vs. 40\%, p =0,02), esta significación no se alcanzó en nuestra serie, ni siquiera cuando se consideraron los DPs grandes.

Son más numerosos los trabajos que describen la relación entre los niveles de glucosa y pH en el DPM y la sensibilidad de la citología, la supervivencia y la tasa de éxito de la pleurodesis [18]. Los valores de glucosa y pH están relacionados con la extensión de las lesiones pleurales. A través del metabolismo de la glucosa se generan iones $\mathrm{H}+$, incapaces de abandonar la cavidad pleural porque sus vías de escape están bloqueadas por las lesiones neoplásicas $(12,13,27)$. Por consiguiente, los DPMs con concentraciones bajas de $\mathrm{pH}$ y glucosa reflejan una mayor carga tumoral en el espacio pleural y, en consecuencia, la probabilidad de hallar una citología positiva aumentaría. Sahn y Good (12) observaron una sensibilidad de la citología del $89 \%$ (17/19) frente a un 37\% (15/40) para los DPMs con un $\mathrm{pH}$ del LP inferior $\mathrm{o} \geq 7,3$ respectivamente $(\mathrm{p}<$ $0,05)$. En el estudio de Rodríguez-Panadero y López-Mejías [13] estos porcentajes también fueron significativamente diferentes en los DPMs con concentraciones de glucosa $<60$ 
$\mathrm{mg} / \mathrm{dl}$ y $\mathrm{pH}<7,3$ (9/10, 90\% sensibilidad), en contraposición a aquellos con valores superiores de estos dos parámetros (20/49, 50\% sensibilidad; $\mathrm{p}<0,03)$. Con posterioridad, otra investigación puso en cuestión estos datos (14), al no hallar diferencias en la sensibilidad de la citología entre DPMs con $\mathrm{pH}$ inferior $(14 / 21,67 \%)$ o $\geq 7,3(10 / 15,67 \%)$. En nuestro estudio, la presencia de concentraciones bajas de $\mathrm{pH}$ y glucosa en LP se relacionó con un mayor número de citologías positivas, pero dichos parámetros se eliminaron en el análisis multivariante en favor de un cociente entre la glucosa del LP y del suero $\leq 0,75$. Probablemente este último evita la distorsión de las cifras de glucosa pleural en sujetos diabéticos.

Ocasionalmente otros datos bioquímicos, distintos del pH y glucosa pleurales, se han asociado con la rentabilidad de la citología; es el caso de la amilasa en el LP. Villena y cols., (28) describen una mayor positividad de las citologías en LPs con amilasa superior al límite normal del suero, 200 U/1 (19/32, $59 \%$ frente a 69/203,34\%; p < 0,01). Aunque nuestra investigación demostró una mayor frecuencia de positividades citológicas en sujetos con DPM y concentraciones elevadas de LDH
(> $1.500 \mathrm{U} / 1)$, amilasa (> $90 \mathrm{U} / \mathrm{l})$ y cociente de proteínas entre LP y suero $>0,77$, como en el caso de la glucosa y $\mathrm{pH}$ pleurales, el modelo de regresión logística sólo observó significación estadística para el cociente entre la glucosa del LP y del suero.

Nuestro estudio tiene algunas limitaciones. Se trata de una serie retrospectiva, en la que no se disponía de segundas y terceras citologías sucesivas en todos los pacientes con una primera negativa. También hemos incluido un grupo substancial de DPs probablemente malignos, sin confirmación histológica definitiva. Sin embargo, se utilizaron criterios muy estrictos, valorando los hallazgos clínicos, las pruebas complementarias realizadas y la evolución del paciente.

En conclusión, la citología continúa siendo una prueba diagnóstica sencilla y de indudable valor en el diagnóstico del DPM. Es aconsejable repetir, de forma inmediata, una segunda toracocentesis para obtener una muestra destinada al análisis citológico, cuando el primero ha sido negativo y se sospecha malignidad. Demorar esta extracción no aumenta la rentabilidad citológica. Los DPMs con un cociente entre la glucosa del LP y del suero $\leq$ 0,75 ofrecen, con más frecuencia, citologías positivas.

\section{Bibliografía}

1. Porcel JM. Manejo práctico del derrame pleural. An Med Interna (Madrid) 2002; 19: 202-8.

2. Rodríguez-Panadero F, Borderas-Naranjo F, López-Mejías J. Pleural metastasic tumours and effusions: Frequency and pathogenic mechanisms in a post-mortem series. Eur Respir J 1989; 2: 366-9.

3. Antunes G, Neville E, Duffy J, Ali N. BTS guidelines for the management of malignant pleural effusions. Thorax 2003; 58: 29-38.

4. Prakash UBS, Reiman HM. Comparison of needle biopsy with cytologic analysis for the evaluation of pleural effusion: Analysis of 414 cases. Mayo Clin Proc 1985; 60: 158-64.

5. Rodriguez-Panadero F, Jansen JP, Astoul P. Thoracoscopy: General overview and place in the diagnosis and management of pleural effusion. Eur Respir J 2006; 28: 409-22.

6. Järvi OH, Kunnas RJ, Laitio MT, Tyrkkö JES. The accuracy and significance of cytologic cancer diagnosis of pleural effusions. (A follow up study of 338 patients). Acta Cytol 1972; 16: 152-8.

7. Starr RL, Sherman ME. The value of multiple preparations in the diagnosis of malignant pleural effusions. A cost-benefit analysis. Acta Cytol 1991; 35: 533-7.

8. Grunze H. The comparative diagnostic accuracy, efficiency and specificity of cytologic techniques used in the diagnosis of malignant neoplasm in serous effusions of the pleural and pericardial cavities. Acta Cytol 1964; 40: 150-63.

9. Nance KV, Shermer RW, Askin FB. Diagnostic efficacy of pleural biopsy as compared with that of pleural fluid examination. Modern Pathol 1991; 4: 320-4.

10. Salyer WR, Eggleston JC, Erozan YS. Efficacy of pleural needle biopsy and pleural fluid cytopathology in the diagnosis of malignant neoplasm involving the pleura. Chest 1975; 67: 536-9.

11. Winkelmann M, Pfitzer P. Blind pleural biopsy in combination with cytology of pleural effusions. Acta Cytol 1981; 24: 373-6.

12. Sahn SA, Good JT. Pleural fluid pH in malignant effusions. Diagnostic, prognostic and therapeutic implications. Ann Intern Med 1988; 107: 345-9.

13. Rodríguez-Panadero F, López-Mejías J. Low glucose and pH levels in malignant pleural effusions. Diagnostic significance and prognostic value in respect to pleurodesis. Am Rev Respir Dis 1989; 139: 663-7.

14. Foresti V, Scolari N, Villa A, Parisio E, De Filippi G, Guareschi G. Malignant pleural effusions: meaning of pleural-fluid $\mathrm{pH}$ determination.

Oncology 1990; 47: 62-4

15. Villena V, Ferrer J, Hernández L, de Pablo A, Pérez E, Rodríguez-Panadero F, et al. Diagnóstico y tratamiento del derrame pleural. Arch Bronconeumol 2006; 42: 349-72.

16. Porcel JM, Light RW. Diagnostic approach to pleural effusion in adults. Am Fam Physician 2006; 73: 1211-20.

17. Motherby H, Nadjari B, Friegel P, Kohaus J, Ramp U, Böcking A. Diagnostic accuracy of effusion cytology. Diagn Cytopathol 1999; 20: 350-7.

18. Antony VB, Loddenkemper R, Astoul P, Boutin C, Goldstraw P, Hott J, et al. Management of malignant pleural effusions. Eur Respir J 2001; 18: 402-19.

19. Sallach SM, Sallach JA, Vásquez E, Schultz L, Kyale P. Volume of pleural fluid required for diagnosis of pleural malignancy. Chest 2002; 122: 1913-7.

20. Renshaw AA, Dean BR, Antman KH, Sugarbaker DJ, Cibas ES. The role of cytologic evaluation of pleural fluid in the diagnosis of malignant mesothelioma. Chest 1997; 111: 106-9.

21. Ahmed S, Shaid RK, Rimawi R, Siddiqui AK, Rossoff L, Sison CP, et al. Malignant pleural effusions in lymphoproliferative disorders. Leuk Lymphoma 2005; 46: 1039-44.

22. Light RW, Erozan YS, Ball WC. Cells in pleural fluid. Arch Intern Med 1973; 132: 854-60.

23. López-Cardozo P. A critical evaluation of 3,000 cytologic analyses of pleural fluid, ascetic fluid and pericardial fluid. Acta Cytol 1966; 10: 455-60.

24. Ong KC, Indumathi V, Poh WT, Ong YY. The diagnostic yield of pleural fluid cytology in malignant pleural effusions. Singapore Med J 2000; 41: 19-23.

25. How SH, Chin SP, Zal AR, Liam CK. Pleural effusions: Role of commonly available investigations. Singapore Med J 2006; 47: 609-13.

26. Jiménez D, Díaz G, Gil D, Cicero A, Pérez-Rodríguez E, Sueiro A, et al. Etiology and prognostic significance of massive pleural effusions. Respir Med 2005; 99: 1183-7.

27. Good JT, Taryle DA, Sahn SA. The pathogenesis of low glucose, low pH malignant effusions. Am Rev Respir Dis 1985; 131: 737-41.

28. Villena V, Pérez V, Pozo F, López-Encuentra A, Echave-Sustaeta J, Arenas $\mathrm{J}$ et al. Amylase levels in pleural effusions: A consecutive unselected series of 841 patients. Chest 2002; 12: 470-4. 